\title{
LIGUE INTERNATIONALE CONTRE LE RHUMATISME
}

The recent International Congress of Rheumatic Diseases held in New York, at which Drs. Hench, Kendall, Slocumb, and Polley announced their discovery of the value of Cortisone in Rheumatoid Arthritis, emphasized the importance of these gatherings sponsored by the International League. Such congresses can only be attended by members of the League and in view of the stimulus to research in the subject given by this new work, the future congresses of the European League in Barcelona in 1951 (last week in May) and of the whole International League in Zurich in 1953, promise to be most important meetings.

Membership of the British Branch of the European League against Rheumatism is open to any doctor interested in the rheumatic diseases on payment of an annual subscription, which is used to defray the British League's subscription to the International League to meet Secretarial and other running expenses and to accumulate a small fund against the day when Great Britain will again become the host for an International meeting.

History.-The Ligue Internationale contre le Rhumatisme was founded in 1929 to act as a co-ordinating body for the International campaign against rheumatism. There had been previous attempts to form such a League, but there had always been difficulty in exchanging information because the same nomenclature has not been used in different countries to describe the various types of rheumatic diseases. Dr. Van Breemen of Amsterdam and Dr. Fortescue Fox of London took the leadership in the formation of the Ligue. But for World War I it would have been started earlier, for in 1913 a proposal was passed to study the rheumatic diseases more methodically in different countries, and a report was to have been given in St. Petersburg in 1917.

In 1925, at a meeting of the International Society of Medical Hydrology in Switzerland, an "International Committee on Rheumatism " was formed which later developed into Le Ligue Internationale contre le Rhumatisme, and in 1929 the first congress of the Ligue was held in Budapest. A medical journal, the Acta Rheumatologica, was started as a medium for the exchange of information and was only "liquidated " after the German invasion of Holland, where it was published. Subsequent congresses were held in Paris and Prague. In 1939 a congress was to have been held in New York, but this had to be postponed for ten years as during World War II all activities of the Ligue were suspended. The records were hidden from the Germans when they occupied Amsterdam, have survived and were shown at the congress held in New York in June, 1949.

Officers.-At the recent congress in New York the following Officers were elected:

President: $\quad$ Prof. EJNAR JARL $\varnothing v$, Copenhagen, Denmark.

President-Elect: $\quad$ Dr. ROBERT STECHER, Cleveland, Ohio, U.S.A.

Assistant to President: Mr. O. DE Bornemann, Copenhagen, Denmark.

Secretary-Treasurer: Dr. W. TEGNER, London, England. 
Councillors of the Ligue Internationale

Dr. A. Michez (Belgium).

Dr. C. H. Traeger (U.S.A.).

Dr. E. JARLøv (Denmark).

Dr. OtTo Steinbrocker (U.S.A.).

Dr. W. S. C. Copeman (England).

Dr. E. Hartung (U.S.A.).

Dr. M.-PierRe WeIl (France).

Dr. E. BOLAND (U.S.A.).

Dr. A. DE ChATel (Hungary).

Dr. Charles Slocumb (U.S.A.).

Dr. H. W. Lunberhuizen (Holland).

Dr. Richard T. SMITH (U.S.A.).

Dr. P. Barcelo (Spain).

Dr. F. Sundelin (Sweden).

Dr. A. Ruiz-Moreno (Argentina).

Dr. K. M. Walthard (Switzerland).

Dr. F. Herrera Ramos (Uruguay).

Dr. A. Lennoch (Czechoslovakia).

Dr. L. M. SAenz (Peru).

Dr. Wallace Graham (Canada).

\section{PAN AMERICAN LEAGUE}

President: $\quad$ Dr. ROBERT STECHER (U.S.A.).

Secretary: Dr. A. RuIz-Moreno (Argentina).

\section{EUROPEAN LEAGUE AGAINST RHEUMATISM}

President: $\quad$ Dr. W. S. C. Copeman, O.B.E. (England).

Secretary: Dr. G. EDSTRöm, Department of Rheumatology, Medical Division, University of Lund, Sweden.

\section{BRITISH BRANCH OF THE EUROPEAN LEAGUE AGAINST RHEUMATISM}

President:

Treasurer:

Secretary:

Executive Committee: Dr. W. S. TEGNER, M.D., M.R.C.P.

Dr. B. S. SCHLESINGER, O.B.E., M.D., F.R.C.P.

Clinics.-The following heads of clinics have been approached and have intimated that members of the League will be welcome. Prior notification of attendance should be given.

\section{JUVENILE RHEUMATISM}

Children's Heart Hospital, Woodlands Way, Wickham, Kent.

Thursday, 10.30 a.m., Dr. R. E. Bonham-Carter (by arrangement).

Canadian Red Cross Memorial Hospital, Maidenhead, Berks.

Dr. E. G. L. Bywaters (by arrangement).

Children's Heart Home, Lancing, Sussex.

Dr. D. de L. McCarthy (by arrangement).

\section{Adult Rheumatism}

BATH

Royal National Hospital for Rheumatic Diseases.

Monday, 10 a.m., In-Patients, Dr. G. D. Kersley; Out-Patients, Dr. L. C. Hill.

Tuesday, 2 p.m., Out-Patients, Dr. J. B. Bennett.

Wednesday, 10 a.m., Out-Patients, Dr. P. W. McKeag.

Thursday, 9.30 a.m., Out-Patients, Dr. G. D. Kersley.

S.W. and Oxford Regional Rheumatism Research Centre.

Daily. Dr. G. D. Kersley. 
Royal Infirmary.

BRISTOL

Friday, 10 a.m., Out-Patients, Dr. G. D. Kersley.

St. Andrew's Brine Baths.

DroITWICH

Tuesday, 9.30 a.m., Dr. J. W. T. Patterson.

Friday, 2 p.m., Dr. H. L. Milles.

Royal Bath Hospital.

HARROGATE

Monday, 10 a.m., Dr. Reah; 2 p.m., Dr. Rutherford.

Tuesday, 10 a.m., Dr. Potter; 2 p.m., Dr. Yeoman.

Wednesday, 10 a.m., Dr. Yeoman; 2 p.m., Dr. Rutherford.

Thursday, 10 a.m., Professor Hartfall.

Friday, 10 a.m., Dr. Wesley-Smith; 2 p.m., Dr. Reah.

\section{LONDON}

Arthur Stanley Institute for Rheumatic Diseases, Middlesex Hospital, Peto Place, Marylebone Road, N.W.1.

Monday, 2.15 p.m., Dr. Oswald Savage.

Tuesday, 10 a.m., Dr. Doris Baker; 2 p.m., Dr. R. Stone; 5 p.m., Dr. H. F. Turney.

Wednesday, 10 a.m., Dr. F. Howitt; 2.15 p.m., Dr. W. S. C. Copeman.

Thursday, 10 a.m., Dr. R. Stone; 5 p.m., Dr. W. S. Tegner.

Friday, 10 a.m., Dr. Ernest Fletcher; 5.30 p.m., Dr. Doris Baker.

Post-Graduate Medical School, Ducane Road, Hammersmith, W.12.

Tuesday, 2 p.m., Out-Patients, Dr. E. G. L. Bywaters.

London Hospital, Department of Physical Medicine, E.1.

Thursday, 10 a.m., Dr. W. S. Tegner.

Friday, 10 a.m.

Royal Free Hospital, Gray's Inn Road, W.C.1.

Wednesday, 1.30 p.m., Dr. Ernest Fletcher.

St. Stephen's Hospital, 369 Fulham Road, S.W.10.

Tuesday, 11.30 a.m., In-Patients, Dr. Philip Ellman.

Wednesday, 10 a.m., Out-Patients, Dr. Philip Ellman.

Thursday, 9.30 a.m., In-Patients, Dr. Francis Bach.

Friday, 9.30 a.m., Out-Patients, Dr. Francis Bach.

West London Hospital, Hammersmith, W.6.

Wednesday, 10.30 a.m., Dr. W. S. C. Copeman, Dr. Oswald Savage.

Westminster Hospital, S.W.1.

Wednesday, 2 p.m., Dr. F. Dudley Hart.

University College Hospital, Department of Physical Medicine, Gower Street, W.C.1.

Tuesday, 10 a.m., Dr. Hugh Burt.

Thursday, 2 p.m.

MANCHESTER

Nuffield Rheumatism Research Centre, Royal Infirmary, Manchester, 13.

Monday, 1.30 p.m., Out-Patients, Dr. J. H. Kellgren.

Wednesday, 10 a.m., In-Patients. 
Royal Infirmary.

\section{WORCESTER}

Wednesday, 9 a.m., Dr. J. W. T. Patterson.

EDINBURGH

Northern General Hospital, Ferry Road.

Monday, 2 p.m., Dr. J. J. R. Duthie.

Wednesday, 2 p.m. (follow up).

Royal Infirmary.

Tuesday, 9.30 a.m., Dr. J. J. R. Duthie.

Friday, 2 p.m. (follow up).

\section{GLASGOW}

Royal Infirmary, 106 Castle Street, Glasgow, C.4.

Tuesday, 9.30 a.m., Dr. J. P. Currie.

Courses.-The following post-graduate courses have been arranged:

(1) March 25-26, 1950. St. Stephen's Hospital, 369 Fulham Road, S.W.10. Particulars through the Fellowship of Medicine.

(2) April 28-30, 1950. Empire Rheumatism Council Spring Week-End Course at the Apothecaries Hall, E.C.4. Particulars from the Empire Rheumatism Council.

Heberden Society Meetings.-Members of the British Branch of the League are invited to the following meetings of the Heberden Society:

(1) The Heberden Round on May 20, 1950, at the Hôpital Cochin, Paris, by Professor Coste, who has extended his personal invitation. The members of the French Branch are also to be invited.

(2) The General Meeting in London on Saturday, December 9, 1950 at 10.15 a.m., at the Meyerstein Theatre, Medical School, Westminster Hospital, Horseferry Road, S.W.1. Discussion on "Endocrine Aspects of the Rheumatic Diseases", by Prof. E. C. Dodds, F.R.S., and Dr. Peter Bishop.

Prior notification of attendance should be sent to The Secretary, Heberden Society, c/o Empire Rheumatism Council, Tavistock House (N), Tavistock Square, W.C.1. 